\title{
Incidence, predictors, and evolution of conduction disorders and atrial arrhythmias after contemporary mitral valve repair
}

\author{
Juan F. Viles-Gonzalez ${ }^{1}$, Alan D. Enriquez ${ }^{2}$, Javier G. Castillo ${ }^{2}$, James O. Coffey ${ }^{1}$, \\ Luciano Pastori ${ }^{3}$, Vivek Y. Reddy ${ }^{2}$, David H. Adams ${ }^{2}$, Valentín Fuster ${ }^{2}$ \\ ${ }^{1}$ University of Miami Miller School of Medicine, Miami, FL, USA \\ ${ }^{2}$ Mount Sinai School of Medicine, New York, NY, USA \\ ${ }^{3}$ Metropolitan Hospital, New York, NY, USA
}

\begin{abstract}
Background: Conduction disorders (CD) and atrial arrhythmias $(A A)$ in the postoperative period of cardiac surgery impede prompt clinical recovery and prolong hospitalization. Mitral valve repair (MVR) has become the treatment of choice for patients with significant valvular regurgitation, but information on $C D$ and $A A$ in this population is scarce.

Methods: Records of consecutive patients undergoing MVR at a single center were reviewed. Patients with a preoperative pacemaker, CD, prior cardiac surgery or concomitant MVR were excluded. A total of 290 patients were included in the final analysis. Electrocardiograms preand post-operatively were analyzed for $C D$ and $A A$.

Results: $C D$ occurred in 69 (23.7\%) patients: 47 (16.2\%) had first degree atrio-ventricular block (AVB), 10 (3.4\%) had Mobitz I, 3 (1.03\%) had Mobitz II, and 9 (3.1\%) complete AVB. Only $6(2.0 \%)$ patients required pacemakers. Univariate predictors of AVB were age, preexisting right bundle branch block (RBBB), mitral valve ring size, and bypass time. The only multivariate predictors of $A V B$ were bypass time and preexisting $R B B B(O R 3.23$ and 1.98 , respectively). The most common AA was atrial fibrillation $13.1 \%$ (38 patients) followed by atrial flutter $2.7 \%$ (8 patients). Multivariate predictors of $A A$ were age and left atrial size (OR 1.85 and 4.2, respectively). Length of stay in patients with $C D$ or $A A$ was prolonged $2.2 \pm 2$ days compared to controls $(p<0.05)$.
\end{abstract}

Conclusions: In this large sample of patients undergoing MVR, we found that bypass time and preexisting $R B B B$ were independent predictors of $C D$; age and left atrial size were independent predictors of $A A$. (Cardiol J 2014; 21, 5: 569-575)

Key words: conduction disorder, atrio-ventricular block, atrial fibrillation, atrial flutter, mitral valve repair, pacemaker

\section{Introduction}

The development of conduction disorders (CDs) has been well described after coronary ar- tery bypass grafting $(\mathrm{CABG})$ and valvular surgery, occurring in 10-20\% of patients with $1-3 \%$ ultimately requiring permanent pacemaker implantation (PPI) [1-6]. In addition, atrial arrhythmias (AAs)

Address for correspondence: Juan F. Viles-Gonzalez, MD, Assistant Professor of Medicine, Clinical Cardiac Electrophysiology, University of Miami Miller School of Medicine, 1120 N.W. 14th Street Miami, FL 33136, USA, tel/fax: (305) 243-5070/5565, e-mail: j.vilesgonzalez@med.miami.edu

Received: 26.11 .2013

Accepted: 09.01.2014 
occur in up to $60 \%$ of patients undergoing cardiac surgery [7-9]. Both postoperative CDs and AAs can have significant clinical consequences, impeding prompt recovery and prolonging hospitalization.

Mitral regurgitation remains a common problem with 40,000 patients undergoing mitral valve surgery each year in the United States [10]. Surgical mitral valve repair (MVR) is the gold standard procedure for patients who require surgery for degenerative mitral valve regurgitation and MVR [11].There are only 2 small studies examining CDs in patients undergoing MVR. The first study included 115 patients and showed an incidence of CDs of $23 \%$ and PPI in 2.6\% [12]. The second study included 391 patients undergoing mitral valve surgery (147 with MVR) and showed CDs in $23.5 \%$ and PPI in $4 \%$ [13]. Understanding the risk factors and natural history of $\mathrm{CD}$ and $\mathrm{AA}$ in patients undergoing MVR may influence both the timing of pacemaker implantation and the strategy to restore sinus rhythm. In light of the scarcity of contemporary data in this area, we sought to investigate the incidence, predictors, and evolution of CDs and AAs after MVR.

\section{Methods}

Data were retrospectively reviewed from all patients with degenerative mitral valve prolapse operated on for mitral regurgitation as the primary indication by a single surgeon (D.H.A.) and his team between March 2008 and December 2010. During this period, 308 consecutive patients underwent MVR. Patients undergoing concomitant cardiac surgery such as tricuspid valve repair (TVR), CABG, patent foramen ovale (PFO) closure, and MAZE, were included in the study population. We excluded from this study patients with permanent or transient cardiac pacing or complete atrioventricular $(\mathrm{AV})$ block before surgery $(\mathrm{n}=4)$, patients undergoing repeat cardiopulmonary bypass $(\mathrm{n}=2)$, patients undergoing septal myomectomy $(\mathrm{n}=2)$, those with mitral stenosis $(n=3)$, and aortic ste$\operatorname{nosis}(\mathrm{n}=7)$.

The study was approved by the local bioethical committee and all patients gave their informed consent.

\section{Surgical technique}

Valve repairs were performed by means of standard reconstructive techniques; sequentially repairing the posterior leaflet, to perform a true-sized annuloplasty with a complete semirigid remodeling ring, and then to repair any residual prolapse of the anterior leaflet or commissures after inspection of the line of closure during testing with saline solution. We typically performed posterior leaflet repair by targeted resection (quadrangular or triangular) of any prolapsing segment, reducing the posterior annular dimension if appropriate and then reconstructing the posterior leaflet. In the setting of excess posterior leaflet tissue, we typically performed a sliding leaflet plasty to reduce the height of the posterior leaflet to $1-1.5 \mathrm{~cm}$ in all segments. In valves with minimal tissue, the prolapse was corrected by limited triangular resections or insertion of artificial polytetrafluoroethylene chordae or loops or by native chordal transfer. Artificial chordae were also often used to reinforce the posterior leaflet resection repair or to displace further the closure line.

All patients (except 7 that underwent minimally invasive surgery) underwent median sternotomy, and cardiopulmonary bypass was established via cannulation of the ascending aorta and right atrium. Cardioplegia and systemic hypothermia was performed according to standard surgical techniques. Temporary epicardial pacemaker leads were implanted during surgery for every patient. Cardiopulmonary bypass time and the size of the annuloplasty ring were recorded for each patient.

\section{Medical record review}

Standardized data forms were used to extract pertinent data from the medical record regarding details of demographics and medical history. Preoperative records were evaluated for each patient in order to examine baseline characteristics, comorbidities, and use of antiarrhythmic or AV nodal blocking medications prior to surgery. Preoperative echocardiograms were also reviewed to assess baseline ventricular function and severity of valvular disease. In order to assess for the development of conduction disturbances, 12 lead electrocardiograms (ECGs) were reviewed independently by 2 cardiologists. Preoperative ECGs were examined for baseline CDs and AAs. All postoperative ECG were reviewed with particular attention paid to the ECG $24 \mathrm{~h}$ after surgery and the ECG at time of discharge. When available, a follow-up ECG 4 or more weeks after the surgery was also reviewed. While AV block was the primary conduction abnormality of interest, bundle branch block, hemi-blocks, and junctional rhythms were all documented. Postoperative ECGs were also reviewed for new AA (atrial tachycardia, atrial fibrillation $[\mathrm{AF}]$, or atrial flutter). All PPIs, cardioversions for AAs, and length of hospitalization for each patient were recorded. 


\section{Statistical analysis}

The following variables were included in the analysis: age, race, gender, height, weight; history of coronary artery disease, hypertension, heart failure, diabetes, pulmonary disease; preoperative medications including calcium-blockers, beta-blockers, amiodarone, digoxin, sotalol, other anti-arrhythmics; electrocardiographic parameters including PR interval, QRS duration, right bundle branch block (RBBB), left bundle branch block (LBBB), left and posterior hemi-blocks; echocardiographic parameters including left ventricular ejection fraction (LVEF), left ventricle (LV) right ventricle (RV); left atrium (LA), right atrium (RA) size and function; procedural characteristics including bypass time, mitral ring size, Maze, TVR, tricuspid ring size. Continuous data were expressed as mean \pm standard deviations, and categorical data were expressed as numbers and percentages. Continuous data were analyzed using Mann-Whitney test, and categorical data were compared using $\chi^{2}$ and Fisher's exact tests, when needed. Potential predictors of $\mathrm{CD}$ and $\mathrm{AA}$ were first identified using univariate logistic regression analyses, with a threshold of $p<0.05$, then included as independent variables in multivariate regression models. Correlation analyses were performed between continuous variables. Multivariate odds ratios (ORs) and corresponding confidence intervals (CIs) were presented for each variable included in the regression model. All statistical analyses were performed using the Statistical Package for the Social Sciences (SPSS Inc.).

\section{Results}

A total of 290 patients were included in the final analysis after the exclusion criteria mentioned above. Baseline clinical characteristics are listed in Table 1. Most patients had prolapse of the posterior leaflet of the mitral valve (75\%), with a mean age $56 \pm 13$ years, $35 \%$ of the patients were females, the mean LVEF was $55 \pm 9 \%$, length of stay was $5 \pm 6$ days, bypass time was $145 \pm 65$ min, mean annuloplasty ring size was $32 \mathrm{~mm}$, successful MVR was achieved in all patients and the in-hospital mortality was zero.

\section{Incidence of conduction disorders and atrial arrhythmias}

CD occurred in 69 (23.7\%) patients: $47(16.2 \%)$ had first degree AV block, 10 (3.4\%) had Mobitz I, $3(1.03 \%)$ had Mobitz II, and 9 (3.1\%) complete AV block. Fifty percent of patients with Mobitz I,
Table 1. Baseline clinical characteristics.

\begin{tabular}{lc}
\hline Demographics & $56 \pm 13$ \\
Age & $35.3 \%$ \\
Female & \\
Leaflet involvement & $75 \%$ \\
Isolated posterior & $6 \%$ \\
Isolated anterior & $19 \%$ \\
Bileaflet & \\
Cardiac co-morbidity & $55 \pm 9$ \\
LVEF [\%] & $6.5 \%$ \\
Moderate to severe MR & $93.5 \%$ \\
Severe MR & $2.3 \%$ \\
Previous MI & $10.8 \%$ \\
Coronary artery disease & \\
\hline
\end{tabular}

LVEF - Left ventricular ejection fraction; MR - mitral regurgitation; $\mathrm{Ml}$ - myocardial infarction

$66 \%$ with Mobitz II and 44\% with complete AV block resolved to first degree AV block. The most common AA was AF $13.1 \%$ (38 patients) followed by atrial flutter $2.7 \%$ (8 patients).

\section{Univariate and multivariate predictors of AV block}

The results of the univariate analysis are presented in Table 2. Univariate predictors of AV block were age, preexisting $\mathrm{RBBB}$, mitral valve ring size, and bypass time. The only multivariate predictors of AV block were bypass time and preexisting RBBB (OR 3.23, 95\% CI 1.45-7.21 and 1.98, 95\% CI 1.11-4.87, $\mathrm{p}<0.05$, respectively). All the other variables including age, race, gender, history of coronary artery disease, hypertension, heart failure, diabetes, pulmonary disease; preoperative medications, ECG parameters, echocardiographic parameters including LVEF, LV, RV, RA size and function and procedural characteristics including mitral ring size, Maze, TVR, and tricuspid ring size, lacked statistical significance.

\section{Univariate and multivariate predictors of atrial arrhythmias}

The results of the univariate analysis are presented in Table 3. Univariate predictors of AA were LA enlargement (RR 4.89, 95\% CI 2.30$-12.27 ; \mathrm{p}<0.05$ ), RA enlargement (RR 1.79, 95\% CI $1.11-4.87 ; \mathrm{p}<0.05)$, age (RR 3.46, 95\% CI 1.30$-7.19 ; \mathrm{p}<0.05$ ), hypertension (RR $1.58,95 \%$ CI 1.07-7.97; $\mathrm{p}<0.05)$, and LV or RV dysfunction (RR 1.87, 95\% CI 1.28-3.15 and 1.72, 95\% CI 1.13-5.58; $\mathrm{p}<0.05$, respectively). 
Table 2. Univariate predictors of conduction disorders.

\begin{tabular}{|c|c|c|c|}
\hline Variables & RR & $95 \% \mathrm{Cl}$ & $\mathbf{P}$ \\
\hline \multicolumn{4}{|l|}{ Demographics } \\
\hline $\operatorname{Age}^{*}$ & 2.43 & $1.22-4.88$ & 0.045 \\
\hline Race & 0.32 & $0.04-3.30$ & 0.345 \\
\hline Gender & 0.48 & $0.14-1.65$ & 0.247 \\
\hline Coronary artery disease & 0.95 & $0.93-1.01$ & 0.901 \\
\hline Hypertension & 2.65 & $0.90-7.15$ & 0.085 \\
\hline Congestive heart failure & 1.02 & $0.22-4.60$ & 0.967 \\
\hline Diabetes & 1.09 & $0.31-3.99$ & 0.988 \\
\hline Pulmonary disease & 0.57 & $0.22-1.15$ & 0.246 \\
\hline Perioperative CCB & 0.59 & $0.28-1.55$ & 0.347 \\
\hline Perioperative beta-blockers & 0.46 & $0.17-1.09$ & 0.090 \\
\hline Perioperative antiarrhythmic & 0.85 & $0.33-2.11$ & 0.788 \\
\hline Perioperative digoxin & 0.32 & $0.03-3.21$ & 0.344 \\
\hline \multicolumn{4}{|l|}{ Electrocardiographic parameters } \\
\hline PR interval & 2.64 & $0.96-6.99$ & 0.059 \\
\hline QRS duration & 1.78 & $0.44-8.01$ & 0.540 \\
\hline Right BBB* & 2.88 & $1.05-8.10$ & 0.040 \\
\hline Left BBB & 1.23 & $0.41-5.62$ & 0.821 \\
\hline Hemiblocks & 1.18 & $0.29-3.98$ & 0.962 \\
\hline \multicolumn{4}{|l|}{ Echocardiographic parameters } \\
\hline Left ventricular ejection fraction & 0.67 & $0.38-1.25$ & 0.845 \\
\hline Left ventricle size & 1.81 & $0.91-3.61$ & 0.122 \\
\hline Right ventricle dysfunction & 0.96 & $0.93-1.06$ & 0.090 \\
\hline Right atrium size & 1.61 & $0.24-3.26$ & 0.112 \\
\hline Left atrium size & 1.64 & $0.33-7.99$ & 0.499 \\
\hline \multicolumn{4}{|l|}{ Operative variables } \\
\hline CPB time* & 6.19 & $2.31-16.01$ & 0.009 \\
\hline Mitral ring size* & 4.01 & $1.40-31.00$ & 0.041 \\
\hline MAZE & 5.03 & $0.44-49.22$ & 0.187 \\
\hline Tricuspid valve repair & 3.99 & 0.95-21 & 0.500 \\
\hline Tricuspid ring size & 0.86 & $0.27-2.68$ & 0.771 \\
\hline
\end{tabular}

*Asterisks identify statistically significant predictors of conduction disorders. Perioperative antiarrhythmics include sotalol and amiodarone; BBB - bundle branch block; CCB — calcium channel blocker; $\mathrm{Cl}$ - confidence interval; CPB — cardiopulmonary bypass time; RR — relative risk

Multivariate predictors of AA were age and LA enlargement (OR 1.85, 95\% CI $1.38-3.15$ and $4.2,95 \%$ CI $2.98-8.11 ; \mathrm{p}<0.05$, respectively). All the other variables including race, gender, history of coronary artery disease, hypertension, heart failure, diabetes, pulmonary disease; preoperative medications, ECG parameters, echocardiographic parameters including LVEF, LV, RV, RA size and function and procedural characteristics including bypass time, mitral ring size, MAZE, TVR, and tricuspid ring size, lacked statistical significance.

\section{Clinical implications}

Length of stay in patients with $\mathrm{CD}$ or AA was significantly prolonged by $2.2 \pm 2$ days compared to controls ( $\mathrm{p}<0.05)$. Out of 46 patients with AA, $16(35 \%)$ patients with AA required electrical cardioversion. Out of 10 patients with Mobitz I, 5 recovered $\mathrm{AV}$ conduction with residual first degree AV block. In 3 patients with Mobitz II, 2 recovered normal $\mathrm{AV}$ conduction and 1 with residual first degree AV block. In 9 patients with complete AV block, 5 recovered AV conduction with residual first degree AV block. The mean time to recovery was $3 \pm 2$ days. Only $6(2 \%)$ patients required pacemakers ( 4 due to $\mathrm{AV}$ block and 2 due to sinus bradycardia). The fatality rate was zero, therefore we could not assess the potential impact of $\mathrm{CD}$ and $\mathrm{AA}$ on mortality in patients undergoing MVR. 
Table 3. Univariate predictors of atrial arrhythmias.

\begin{tabular}{|c|c|c|c|}
\hline Variables & $\mathbf{R R}$ & $95 \% \mathrm{Cl}$ & $\mathbf{P}$ \\
\hline \multicolumn{4}{|l|}{ Demographics } \\
\hline $\mathrm{Age}^{*}$ & 1.44 & $1.12-6.70$ & 0.039 \\
\hline Race & 0.99 & $0.86-1.13$ & 0.911 \\
\hline Gender & 0.98 & $0.86-1.12$ & 0.850 \\
\hline Coronary artery disease & 1.15 & $0.93-1.32$ & 0.130 \\
\hline Hypertension* & 1.50 & $1.29-1.75$ & 0.009 \\
\hline Congestive heart failure & 1.02 & $0.89-1.17$ & 0.867 \\
\hline Diabetes & 1.09 & $0.31-3.99$ & 0.988 \\
\hline Pulmonary disease & 0.79 & $0.52-1.22$ & 0.346 \\
\hline Perioperative CCB & 0.76 & $0.37-1.59$ & 0.547 \\
\hline Perioperative beta-blocker & 0.46 & $0.17-1.39$ & 0.102 \\
\hline Perioperative antiarrhythmics & 0.39 & $0.23-2.88$ & 0.178 \\
\hline Perioperative digoxin & 0.82 & $0.66-1.03$ & 0.103 \\
\hline \multicolumn{4}{|l|}{ Electrocardiographic parameters } \\
\hline PR interval & 0.78 & $0.16-6.82$ & 0.544 \\
\hline QRS duration & 1.80 & $0.18-6.02$ & 0.968 \\
\hline Right BBB & 0.88 & $0.15-4.10$ & 0.400 \\
\hline Left BBB & 1.20 & $0.37-3.68$ & 0.921 \\
\hline Hemiblocks & 1.38 & $0.29-4.98$ & 0.532 \\
\hline \multicolumn{4}{|l|}{ Echocardiographic parameters } \\
\hline Left ventricular ejection fraction* & 2.67 & $1.08-7.25$ & 0.045 \\
\hline Left ventricle size & 0.81 & $0.27-2.61$ & 0.799 \\
\hline Right ventricle dysfunction* & 2.96 & $1.19-7.06$ & 0.029 \\
\hline Right atrium size* & 5.41 & $1.09-22.36$ & 0.032 \\
\hline Left atrium size* & 1.20 & $1.06-1.43$ & 0.042 \\
\hline \multicolumn{4}{|l|}{ Operative variables } \\
\hline CPB time & 0.96 & $0.93-1.01$ & 0.090 \\
\hline Mitral ring size & 0.81 & $0.33-3.01$ & 0.741 \\
\hline MAZE & 1.63 & $0.44-9.22$ & 0.486 \\
\hline Tricuspid valve repair & 0.49 & $0.14-1.65$ & 0.245 \\
\hline Tricuspid ring size & 1.86 & $0.27-8.68$ & 0.574 \\
\hline
\end{tabular}

*Asterisks identify statistically significant predictors of atrial arrhythmias. Perioperative antiarrhythmics include sotalol and amiodarone; $\mathrm{BBB}$ - bundle branch block; CCB — calcium channel blocker; $\mathrm{Cl}$ - confidence interval; CPB — cardiopulmonary bypass time; RR - relative risk

\section{Discussion}

Understanding the risk factors and natural history of CD and AA in patients undergoing MVR may influence the perioperative management of temporary pacemakers, decision of pacemaker implantation and prophylactic or therapeutic strategies to maintain or restore normal sinus rhythm. In view of the paucity of contemporary data in this area, we sought to investigate the incidence, predictors, and evolution of CDs and AAs after MVR. The main findings of our study in patients undergoing surgical MVR are: 1) a significant proportion of patients undergoing MVR with CD had improved $\mathrm{AV}$ conduction (55\%) within $3 \pm 2$ days; 2 ) only
$2.4 \%$ patients required pacemakers; 3 ) bypass time and preexisting RBBB were predictors for the development of $\mathrm{CD}$ after MVR; 4) the incidence of $\mathrm{AA}$ was low, the most common AA was AF followed by atrial flutter, and $35 \%$ patients required electrical cardioversion; 5) predictors of AA were age and LA size; 6) hospitalization in patients with $\mathrm{CD}$ or $\mathrm{AA}$ was prolonged $2.2 \pm 2$ days compared to controls.

Compared to previous studies the incidence of $\mathrm{CD}$ in our cohort was marginally lower. Meimoun et al. [12] reported on the incidence of AV block in patients undergoing MVR over a decade ago. The investigators observed an incidence of $6 \%$ of complete AV block, $3 \%$ of second degree AV block and $14 \%$ first degree AV block. A lower number of 
patients in their series recovered AV conduction (44\% vs. 55\% in our cohort). Another significant difference was higher mortality on their series: it was $1.7 \%$ as compared to zero in our patient population. The need for PPI was similar to our study; 3 (2.6\%) patients had PPI an average of 18 days after surgery. In all patients, it was due to persistent complete AV block, which occurred immediately after surgery. This is in contrast to our study that had $2 \%$ need for PPI, 4 due to AV block and 2 due to sinus bradycardia. Since the incidence of PPI was low, we could not extract any meaningful conclusion regarding potential risk factors for PPI.

Based on previously published data, there are no strong predictors for postoperative AV block in patients undergoing MVR. Nonetheless, we found that age, preexisting RBBB, mitral valve ring size, and bypass time to be associated with the development of $\mathrm{CD}$. Only bypass time and preexisting $\mathrm{RBBB}$ were independent predictors of postoperative $\mathrm{CD}$. This remains in contrast to another study that found a modest interaction between risk of postoperative AV block and body temperature during cardiopulmonary bypass time. Our study was not designed to understand the mechanisms leading to postoperative $\mathrm{CD}$ but it could be speculated that variables related to intraoperative "ischemic time" like body temperature or duration of cardiopulmonary bypass time, and degree of mechanical disruption by a larger ring size, could cause reversible injury to the AV conduction system followed by spontaneous recovery in most patients. Preexisting abnormalities in the right bundle probably increase the propensity of the patient to develop CD after they sustain mechanical trauma to the left bundle system during MVR. Moreover, the characteristics of the conduction system, which differ from those of cardiomyocytes, make it more susceptible to ischemia during cardioplegia, which may account for this clinical observation.

Atrial fibrillation, the most common arrhythmia after cardiac surgery, occurs with an incidence after valvular surgery that exceeds that after coronary artery bypass surgery. In comparison to the $17 \%$ to $33 \%$ incidence of postoperative $\mathrm{AF}$ following coronary bypass surgery, AF develops after cardiac valvular surgery in $38 \%$ to $64 \%$ of patients [14]. The frequency of AA in our study was significantly lower than in prior reports. Similar studies found that as many as $36 \%$ of the patients undergoing MVR would develop AF [9] compared to only $13.1 \%$ in our analysis. In this study, the investigators found that advanced age, mitral stenosis, LA enlargement, use of systemic hypothermia, and a history of cardiac surgery were independent predictors of postoperative AF included. However, only advanced age, mitral stenosis, and LA enlargement were confirmed as independent risk factors in a validation cohort. Our analysis in patients who underwent exclusively MVR revealed that atrial enlargement, hypertension, and LV or $\mathrm{RV}$ dysfunction were predictors of all AA; but like in the former study only age and LA enlargement remained statistically significant as independent predictors. It must be underscored that a fundamental difference between this 2 studies is the fact that we excluded patients with mitral stenosis and prior cardiac surgery.

\section{Limitations of the study}

The main limitation of this study arises from the retrospective nature. In addition, ours is a high-volume mitral valve reference center, and this series represents a single surgeon's highly specialized team results; hence, we cannot exclude the possibility of confounders or selection bias as all the patients in our cohort. Therefore, our conclusions should be considered strictly hypothesis generating. Also, the low event rate of PPI makes it difficult to extract any conclusion regarding the impact of risk factors for CD on rates of PPI. The inclusion of patients undergoing concomitant TVR, CABG, PFO closure, and MAZE introduces major confounders that could have an impact on the results of our analysis. Finally, our study did not include any long-term follow-up, precluding any determination of whether postoperative CD and AA were associated with long-term morbidity and/or mortality. Nevertheless, the strength of the data comes from the fact that this is a large sample of patients undergoing MVR. While we identified risk factors for the development of $\mathrm{CD}$ and $\mathrm{AA}$, validation of these findings in a prospective fashion is warranted.

\section{Conclusions}

In this large sample of patients undergoing MVR, the incidence of $\mathrm{CD}, \mathrm{AA}$ and the need for pacemaker are lower than in prior reports of mitral valve surgery. We found that bypass time and preexisting RBBB were independent predictors of $\mathrm{CD}$; age and LA size were independent predictors of AA. The implications of our findings related to risk factors and natural history of $\mathrm{CD}$ and $\mathrm{AA}$ in patients undergoing MVR, may influence the perioperative management of these patients. Importantly, measures directed at preventing or promptly treating 
both $\mathrm{CD}$ and $\mathrm{AA}$ appear to be warranted since both increase utilization of resources (electrical cardioversion and length of stay). The extent to which the variables reported in this study predict the occurrence of $\mathrm{CD}$ and AA should be evaluated prospectively.

\section{Conflict of interest: None declared}

\section{References}

1. Baerman JM, Kirsh MM, de Buitleir M et al. Natural history and determinants of conduction defects following coronary artery bypass surgery. Ann Thoracic Surgery, 1987; 44: 150-153.

2. Keefe DL, Griffin JC, Harrison DC, Stinson EB. Atrioventricular conduction abnormalities in patients undergoing isolated aortic or mitral valve replacement. Pacing and clinical electrophysiology. PACE, 1985; 8: 393-398.

3. Gundry SR, Sequeira A, Coughlin TR, McLaughlin JS. Postoperative conduction disturbances: A comparison of blood and crystalloid cardioplegia. Ann Thoracic Surgery, 1989; 47: 384-390.

4. Merin O, Ilan M, Oren A et al. Permanent pacemaker implantation following cardiac surgery: Indications and long-term follow-up. Pacing and clinical electrophysiology. PACE, 2009; 32: 7-12.

5. Bagur R, Manazzoni JM, Dumont E et al. Permanent pacemaker implantation following isolated aortic valve replacement in a large cohort of elderly patients with severe aortic stenosis. Heart, 2011; 97: 1687-1694.

6. Ferrari AD, Sussenbach CP, Guaragna JC et al. Atrioventricular block in the postoperative period of heart valve surgery: Inci- dence, risk factors and hospital evolution. Revista Brasileira de Cirurgia Cardiovascular, 2011; 26: 364-372.

7. Maisel WH, Rawn JD, Stevenson WG. Atrial fibrillation after cardiac surgery. Ann Internal Med, 2001; 135: 1061-1073.

8. Creswell LL, Schuessler RB, Rosenbloom M, Cox JL. Hazards of postoperative atrial arrhythmias. Ann Thoracic Surg, 1993; 56: 539-549.

9. Asher CR, Miller DP, Grimm RA, Cosgrove DM, 3rd, Chung MK. Analysis of risk factors for development of atrial fibrillation early after cardiac valvular surgery. Am J Cardiol, 1998; 82: 892-895.

10. Glower DD. Surgical approaches to mitral regurgitation. J Am Coll Cardiol, 2012; 60: 1315-1322.

11. Bonow RO, Carabello BA, Chatterjee K et al. 2008 Focused update incorporated into the ACC/AHA 2006 guidelines for the management of patients with valvular heart disease: A report of the American College of Cardiology/American Heart Association Task Force on Practice Guidelines (Writing Committee to Revise the 1998 Guidelines for the Management of Patients With Valvular Heart Disease): Endorsed by the Society of Cardiovascular Anesthesiologists, Society for Cardiovascular Angiography and Interventions, and Society of Thoracic Surgeons. Circulation, 2008; 118: e523-e661.

12. Meimoun P, Zeghdi R, D'Attelis $\mathrm{N}$ et al. Frequency, predictors, and consequences of atrioventricular block after mitral valve repair. Am J Cardiol, 2002; 89: 1062-1066.

13. Berdajs D, Schurr UP, Wagner A, Seifert B, Turina MI, Genoni M. Incidence and pathophysiology of atrioventricular block following mitral valve replacement and ring annuloplasty. Eur J Cardio-thoracic Surgery, 2008; 34: 55-61.

14. Aranki SF, Shaw DP, Adams DH et al. Predictors of atrial fibrillation after coronary artery surgery current trends and impact on hospital resources. Circulation, 1996; 94: 390-397. 